\title{
A wireless MIMO CPM system with blind signal separation for incoherent demodulation
}

\section{O. Weikert and U. Zölzer}

Helmut Schmidt University - University of the Federal Armed Forces Hamburg, Department of Signal Processing and Communications, Holstenhofweg 85, 22043 Hamburg, Germany

\begin{abstract}
A multiple-input multiple-output (MIMO) wireless transmission system with continuous phase modulation (CPM) is considered. A novel MIMO CPM receiver with incoherent modulation is presented. The incoherent demodulation of CPM allows an uncomplicated handling of a frequency offset compared to coherent approaches requiring exact knowledge of the carrier frequency. Blind signal separation (BSS) is applied in the proposed MIMO CPM receiver to separate the signals without any knowledge of the MIMO channel. The BSS permits the demodulation of each separated signal by an incoherent CPM demodulator. For bandwidth efficient transmission partial response CPM and nonbinary modulation is applied. The applicability of the proposed system is verified by simulation results.
\end{abstract}

\section{Introduction}

Continuous phase modulation (CPM) offers a remarkable amount of benefits. The constant envelope is well suitable for high frequency components. Incoherent demodulation of CPM allows an uncomplicated handling of frequency offsets compared to coherent approaches requiring exact knowledge of the carrier frequency. Multiple-input multiple-output (MIMO) systems provide higher data rates that data-demanding applications require. Applying continuous phase modulation in wireless MIMO systems provides the opportunity to combine the benefits of higher data rates with the capability of incoherent demodulation. As CPM belongs to the class of non-linear modulation schemes the separation of the mixed receive signals is a well-known challenge.

Training based channel estimation is not directly applicable for MIMO systems with continuous phase modulation. Regarding the training symbols for linear modulation schemes the principle of superposition holds. This is not the case for nonlinear modulation schemes like continuous phase

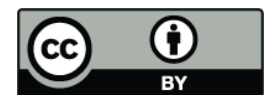

Correspondence to: O. Weikert (oomke.weikert@hsu-hamburg.de) modulation. At the MIMO CPM receiver each receive antenna receives a linear superposition of all transmit signals. However these receive signals are not a linear superposition of the training symbols. The training symbols have to be inserted into the transmit signal prior to the nonlinear modulation. The circumstance is explained in detail later on.

Binary CPM signals can be represented as a sum of a finite number of amplitude modulated pulses (Laurent, 1986). Using the decomposition in Laurent (1986) a layered MIMO system with binary CPM signals is proposed in Zhao and Giannakis (2005). The system in Zhao and Giannakis (2005) is a coherent demodulator which requires exact knowledge of the carrier frequency. The demodulation has a very high complexity due to the representation of CPM signals by amplitude modulated pulses. In the MIMO CPM receiver in Zhao and Giannakis (2005) the perfect knowledge of the MIMO channel is assumed. The practical realization of any MIMO CPM system requiring knowledge of the MIMO channel in the receiver is questionable as training based channel estimation is not applicable for MIMO CPM systems.

Space-Time Codes for MIMO CPM are proposed with its design criteria in Zhang and Fitz (2003), a soft decision iterative receiver is described (Zhang and Fitz, 2002). The system in Zhang and Fitz (2002) is a coherent demodulator which requires exact knowledge of the carrier frequency. The MIMO CPM receivers with Space-Time Codes have a high complexity.

In this paper a MIMO CPM receiver with an incoherent demodulation of the CPM signals is presented. Blind signal separation (BSS) is applied in the MIMO CPM receiver to separate the transmitted signals. A carrier frequency mismatch in the receiver can be handled by the BSS. The BSS permits the demodulation of each separated signal by an incoherent CPM demodulator. On the separated signals classical algorithms for timing and carrier frequency synchronization can be applied. In the proposed MIMO CPM receiver neither any knowledge of the MIMO channel nor the exact carrier frequency is required. The system can cope with any modulation filter and is not restricted to binary modulation. For bandwidth efficient transmission partial response

Published by Copernicus Publications on behalf of the URSI Landesausschuss in der Bundesrepublik Deutschland e.V. 

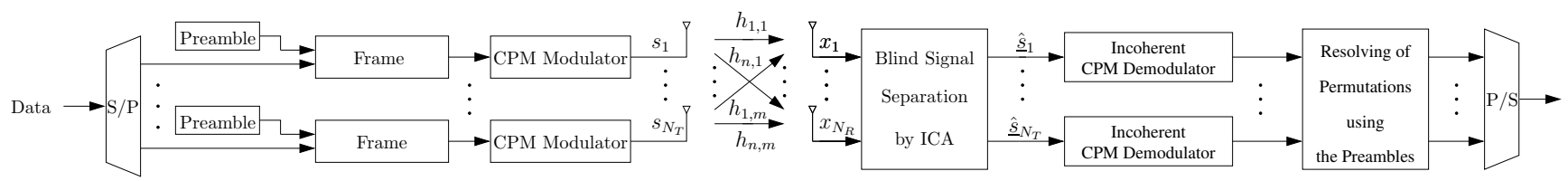

Fig. 1. Proposed wireless MIMO CPM system with incoherent demodulation.

CPM and non-binary modulation is applied. As the order of the transmitted signals cannot be determined by blind signal separation the permutations are resolved by transmission of preamble symbols.

The paper is organized as follows. The MIMO system and the MIMO channel model with the frequency offset introduced is described in Sect. 2. The MIMO receiver for continuous phase modulation with blind signal separation and incoherent demodulation is presented in Sect. 3. Simulation results are given in Sect. 4. A summary and conclusion marks can be found in Sect. 5 .

\section{System description}

\subsection{MIMO CPM transmitter}

We consider a MIMO wireless system with $N_{T}$ transmit and $N_{R}$ receive antennas (see Fig. 1). The serial bit stream is split into $N_{T}$ parallel substreams, one for every transmit antenna. The substreams are organized in frames. Each frame of length $N_{F}$ consists of $N_{I}$ payload bits and $N_{P}$ preamble bits. The preamble sequences are orthogonal to each other. The CPM modulators generating the transmit signals are described in the next section. The signal substreams are subsequently transmitted over the $N_{T}$ antennas at the same time. The symbol transmitted by antenna $m$ at time instant $k$ is denoted by $s_{m}(k)=e^{j \varphi_{m}(k)}$. The transmitted symbols are arranged in vector

$$
\begin{aligned}
s(k) & =\left[s_{1}(k), \ldots, s_{N_{T}}(k)\right]^{T} \\
& =\left[e^{j \varphi_{1}(k)}, \ldots, e^{j \varphi_{N_{T}}(k)}\right]^{T}
\end{aligned}
$$

of length $N_{T}$, where $(\cdot)^{T}$ denotes the transpose operation.

\subsection{CPM modulator}

With continuous phase modulation (CPM) a constant signal envelope with a continuous phase are obtained. The CPM signal is described by Anderson et al. (1986)

$$
s(t)=e^{j\left(\varphi(t)+\varphi_{0}\right)}
$$

with an arbitrary phase offset $\varphi_{0}$ and the instantaneous phase

$$
\varphi(t)=2 \pi h \int_{0}^{t} \sum_{i=0}^{l} d(i) g(\tau-i T) d \tau
$$

with modulation index $h$, the transmit filter $g(t)$, the symbols $d(l)$ and the symbol interval $T$. The discrete CPM modulator is given in Fig. 2. The symbols $d(l)$ are taken from the $M$-ary alphabet $d(l) \in\{ \pm 1, \pm 3, \ldots, \pm(M-1)\}$ with $M$ even.

Before pulse shaping the symbols are upsampled by the factor $S P S$ specifying the number of samples per symbol. Between two successive symbols $S P S-1$ zeros are inserted. The transmit filter

$$
g(k)= \begin{cases}\frac{1}{2 L \cdot S P S}\left(1-\cos \left(\frac{2 \pi k}{L \cdot S P S}\right)\right) & 0 \leq k \leq L \cdot S P S \\ 0 & \text { elsewise }\end{cases}
$$

is a raised cosine impulse (L-RC) (Anderson et al., 1986) with the length of the impulse $L$ in symbol intervals. The transmit filter fulfills the normalization condition

$$
\sum_{k=0}^{L \cdot S P S} g(k)=\frac{1}{2} .
$$

A full response CPM has a transmit filter of length $L=1$. With overlapping transmit pulses that is a transmit filter with $L>1$ the scheme is denoted as partial response. By introducing symbol interferences a higher bandwidth efficiency is obtained (Anderson et al., 1986). After pulse shaping the signal is multiplied by $2 \pi h$ and integrated.

\subsection{MIMO channel}

The non-frequency selective MIMO channel can be described by a complex channel matrix

$$
\mathbf{H}=\left[\begin{array}{ccc}
h_{1,1} & \cdots & h_{1, N_{T}} \\
\vdots & \ddots & \vdots \\
h_{N_{R}, 1} & \cdots & h_{N_{R}, N_{T}}
\end{array}\right]
$$

of the dimension $N_{R} \times N_{T}$. We suppose that the channels remain constant over the transmission of a frame and vary independently from frame to frame (block fading channel). The elements $h_{n, m}$ are complex random variables with a Gaussian distributed real and imaginary part, zero mean and variance $\sigma^{2}$. The element $h_{n, m}$ includes also the direct component with the amplitude $p_{n, m}$ and the power $p_{n, m}^{2}=c_{R} \cdot \sigma^{2}$, where $c_{R}$ is the Rice factor.

The channel energy is normalized by the condition $E\left\{\left|h_{n, m}\right|^{2}\right\}=p_{n, m}^{2}+\sigma^{2}:=1$. We assume additive white Gaussian noise (AWGN) with zero mean and variance $\sigma_{n}^{2}$ per receive antenna. 


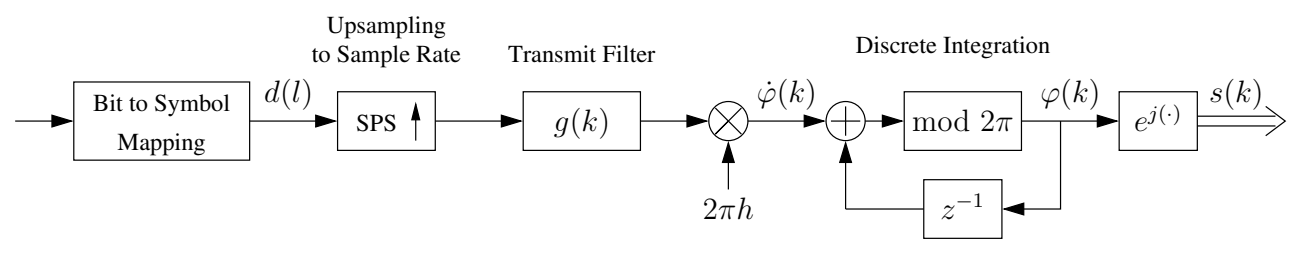

Fig. 2. CPM modulator.

\subsection{Frequency offset}

The discrepancy of the carrier frequency at the receiver from the one used at the transmitter is specified by the frequency offset $\Delta f$. The impact is described in the continuous case by the multiplication of the signal by $e^{j 2 \pi \Delta f t}$. By sampling of the signal the frequency offset $\Delta f$ is related to the sampling frequency $f_{A}=1 / T_{A}$ and the normalized frequency offset $\Delta F=\Delta f / f_{A} \in[-0.5,0.5]$ is introduced. Due to the sampling theorem the determinable range of the frequency offset is limited to $\left[-\frac{f_{A}}{2},+\frac{f_{A}}{2}\right]$.

\subsection{Receive signals}

The symbol received by antenna $n$ at time instant $k$ is denoted by $x_{n}(k)$. Calculation of $x_{n}(k)$ can be expressed as a discrete sum

$$
x_{n}(k)=e^{j 2 \pi \Delta F k} \sum_{m=1}^{N_{T}} h_{n, m} e^{j\left(\varphi_{m}(k)+\varphi_{0}\right)}+n(k),
$$

with $\varphi_{m}(k)$ as a function of the symbols $d(l)$ where $d(l)$ denote the symbols in front of the CPM modulator. $n(k)$ is the additive white Gaussian noise at time instant $k$. As the receive signals $x_{n}(k)$ depend in a nonlinear manner on the symbols $d(l)$, linear methods for channel estimation are not applicable.

The symbols received by the $N_{R}$ antennas are arranged in a vector $\boldsymbol{x}(k)=\left[x_{1}(k), \ldots, x_{N_{R}}(k)\right]^{T}$ of length $N_{R}$, which can be expressed with Eqs. (1), (6) and $\boldsymbol{n}(k)$ as noise vector of length $N_{R}$ as

$$
\boldsymbol{x}(k)=e^{j 2 \pi \Delta F k} \mathbf{H} \mathbf{s}(k)+\boldsymbol{n}(k) .
$$

\section{MIMO CPM receiver with incoherent demodulation}

The MIMO receiver for continuous phase modulation in Fig. 1 uses blind signal separation to estimate and separate the independent components, i.e. the transmitted signals. The blind signal separation is performed at the sampling rate $f_{A}$ which is by the factor SPS higher than the symbol rate. A frequency offset is supposed. Each separated signal corresponds to one of the $N_{T}$ transmitted signals. The BSS permits the demodulation of each separated signal by an incoherent CPM demodulator. The incoherent CPM demodulator does not require any knowledge about the frequency offset. As the order of the transmitted signals cannot be determined by blind signal separation preamble sequences are transmitted to resolve the permutations. Subsequently the transmitted signals are extracted from the frames of the $N_{T}$ parallel substreams.

\subsection{Blind signal separation}

\subsubsection{Whitening}

The whitening transformation

$$
\mathbf{z}=\mathbf{V} \mathbf{x}=\mathbf{V H} \underline{\mathbf{s}}
$$

with

$$
\begin{aligned}
\underline{\mathbf{s}}(k) & =e^{j 2 \pi \Delta F k} \mathbf{s}(k) \\
\underline{s}_{m}(k) & =e^{j 2 \pi \Delta F k} s_{m}(k)
\end{aligned}
$$

and the linear transformation matrix $\mathbf{V}$ (Hyvärinen et al., 2001) of dimension $N_{T} \times N_{R}$ decorrelates the observed mixtures $\mathbf{x}$. The number of observed mixtures $N_{R}$ is reduced to the number of transmitted signals $N_{T}$.

\subsubsection{Independent Component Analysis}

Independent Component Analysis (ICA) separates the observed mixtures $\mathbf{z}$ of the mixing model

$$
\mathbf{z}=\mathbf{V H} \underline{\mathbf{s}}
$$

into the symbols $\underline{\mathbf{s}}$, called independent components (IC), while the mixing matrix $\mathbf{V H}$ is unknown.

Using ICA the following assumptions hold (Hyvärinen et al., 2001):

- ICs must have nongaussian distributions (e.g. leptokurtic or platykurtic)

- ICs have to be statistically independent

- ICs are assumed to have zero mean

- The number of observed mixtures has to be at least equal to the number of ICs

The below-mentioned ambiguities regarding the estimated ICs remain (Hyvärinen et al., 2001): 


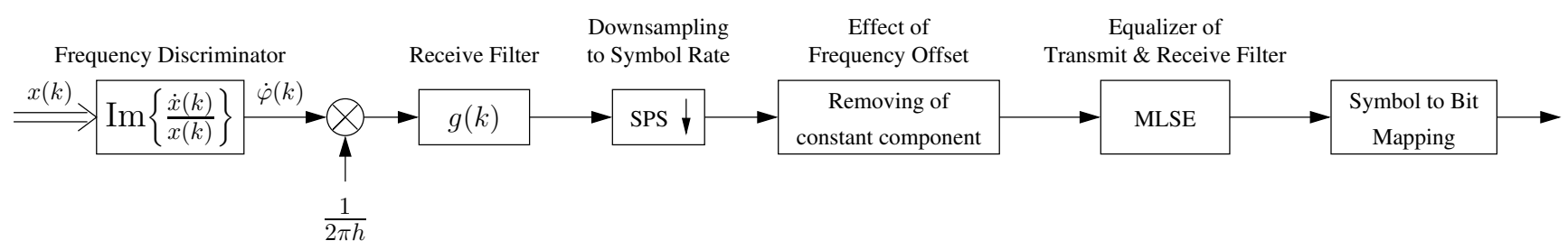

Fig. 3. Incoherent CPM demodulator.

- The estimated ICs can be arbitrarily permuted

- The phases of the estimated ICs are unknown

- The variances (energy) of the estimated ICs can not be determined

Ordinary complex ICA algorithms like JADE (Cardoso and Souloumiac, 1993; Hyvärinen et al., 2001) are applied to obtain estimates of the separated signals $\hat{s}_{m}(k)$. The ICA algorithms compute the unitary matrix $\mathbf{W}^{H}$ to calculate an estimate

$$
\underline{\hat{\mathbf{s}}}=\mathbf{W}^{H} \mathbf{z}=\mathbf{W}^{H} \mathbf{V H} \underline{\mathbf{s}}
$$

of the independent components. As the channel is assumed to be a block fading channel the received data is processed in frames. Recalling the restrictions of ICA, the estimated ICs are arbitrary permuted.

\subsection{Incoherent CPM demodulator}

The incoherent CPM demodulator is given in Fig. 3. The frequency discriminator demodulates the receive signal. The receive filter corresponds to the transmit filter $g(k)$ in Eq. (4). The receive filter is followed by the downsampling to symbol rate. The impact of the frequency offset can be eliminated by removing the signals constant component. The constant component is estimated and subtracted from the signal. Based on the receive signal $x(t)=s(t) \cdot e^{j 2 \pi \Delta f t}$ the signal after the frequency discriminator can be written as

$$
\begin{aligned}
\operatorname{Im}\left\{\frac{\dot{x}(t)}{x(t)}\right\} & \\
& =\operatorname{Im}\left\{\frac{j \dot{\varphi}(t) s(t) e^{j 2 \pi \Delta f t}+s(t) j 2 \pi \Delta f e^{j 2 \pi \Delta f t}}{s(t) e^{j 2 \pi \Delta f t}}\right\} \\
& =\dot{\varphi}(t)+2 \pi \Delta f
\end{aligned}
$$

with $\dot{\varphi}(t)=2 \pi h \sum_{i=0}^{l} d(i) g(t-i T)$. The frequency offset causes a constant component in the demodulated signal. In the discrete case the signal after the frequency discriminator follows to

$$
\operatorname{Im}\left\{\frac{\dot{x}(k)}{x(k)}\right\}=\dot{\varphi}(k)+2 \pi \Delta F
$$

with the normalized frequency offset $\Delta F$.
Using a partial response CPM with overlapping transmit pulses the received signal has to be equalized. For the equalization of the overlapping transmit pulses a maximum likelihood sequence estimation (MLSE) implemented as a Viterbi equalizer is used. For a full response CPM no equalizer is needed since no overlapping transmit pulses are existing.

\subsection{Resolving permutations}

The ambiguities left by blind signal separation are solved by orthogonal preamble sequences (Weikert and Zölzer, 2006). The preambles are the orthogonal rows of a Hadamard matrix $\mathbf{S}_{P}$. Hadamard matrices are binary orthogonal matrices. To ensure the orthogonality the preamble length $N_{P}$ per transmit antenna should be $N_{P} \geq N_{T}$. The received preambles are extracted and inserted line by line in the matrix $\hat{\mathbf{S}}_{P}$. A hard decision is applied, subsequently the preamble matrix is labeled as $\tilde{\mathbf{S}}_{P}$. The unitary cross correlation matrix

$$
\mathbf{R}_{\tilde{\mathbf{S} S}}=\frac{1}{N_{P}} \tilde{\mathbf{S}}_{P} \mathbf{S}_{P}^{H}
$$

where $(\cdot)^{H}$ denotes the complex-conjugate (Hermitian) transpose, will be an identity matrix if no ambiguities exist. Elsewise the ambiguities are resolved by

$$
\mathbf{s}(k)=\mathbf{R}_{\tilde{\mathbf{S}} \mathbf{S}}^{-1} \cdot \hat{\mathbf{s}}(k)=\mathbf{R}_{\tilde{\mathbf{S}} \mathbf{S}}^{H} \cdot \hat{\mathbf{s}}(k) .
$$

\section{Simulation results}

The simulations are performed using a non-frequency selective MIMO system with a line of sight between the transmit and receive antennas. Thereby the Rice coefficient is chosen to be $c_{R}=1$. For the MIMO CPM system $M=4$-ary symbols, a modulation index $h=0.5$ and $S P S=8$ samples per symbol are chosen. The blind signal separation is performed with the JADE algorithm, $N_{F}=100$ symbols per frame i.e. 800 samples per frame. For 2 and 4 transmit antennas $N_{P}=2$ and 4 preamble symbols per antenna are used respectively. The discrete differentiation is performed by a FIR filter of order 3 .

In Figs. 4 and 5 one can see the Bit Error Rate (BER) versus the Signal-to-Noise-Ratio (SNR) for the MIMO CPM system with incoherent demodulation. The results are shown for a normalized frequency offset of $\Delta F=0.05$ and for zero 


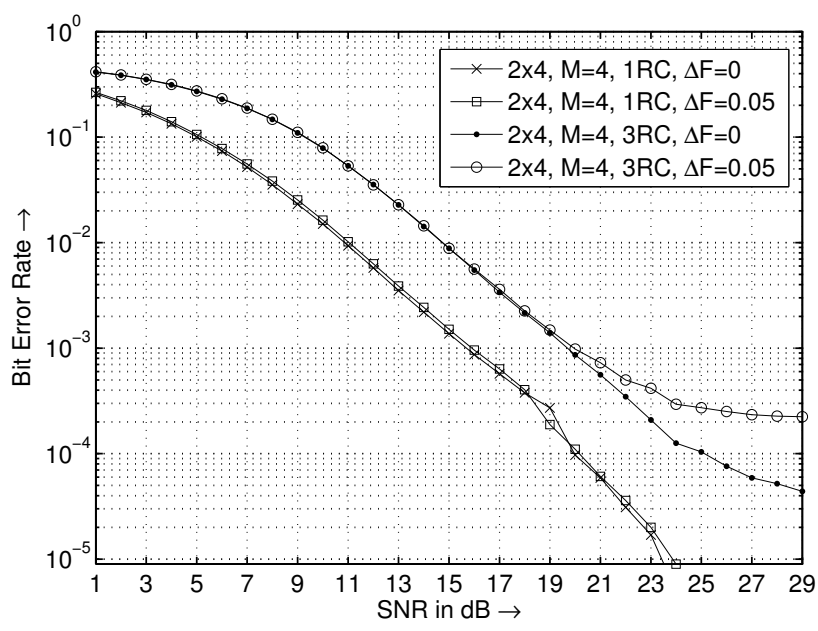

Fig. 4. Bit Error Rate (BER) versus the Signal-to-Noise-Ratio (SNR) for the MIMO CPM system with $N_{T}=2$ transmit and $N_{R}=4$ receive antennas.

frequency offset. For bandwidth efficient transmission a partial response MIMO CPM (3RC) with a raised cosine transmit filter of length 3 (in symbol intervals) is used. Furthermore the results for a full response MIMO CPM (1RC) are shown. In Fig. 4 the results with $N_{T}=2$ transmit and $N_{R}=4$ receive antennas $(2 \times 4)$ are presented. Figure 5 shows the results with $N_{T}=4$ transmit and $N_{R}=6$ receive antennas $(4 \times 6)$. In comparison to a GSM system with binary symbols and a single transmit antenna, the data rate is increased by a factor of eight for the MIMO CPM system with 4 transmit antennas and $M=4$-ary symbols.

The presented MIMO CPM system achieves a Bit Error Rate less than $1 \cdot 10^{-5}$ for a full response MIMO CPM (1RC) with a SNR higher than $25 \mathrm{~dB}$ even in the presence of a frequency offset. An increased frequency offset results in a slightly increased Bit Error Rate. A bandwidth efficient partial response MIMO CPM (3RC) requires a $5 \mathrm{~dB}$ increased SNR to achieve a Bit Error Rate of $5 \cdot 10^{-3}$ compared to the full response MIMO CPM.

\section{Conclusions}

We proposed a semi-blind MIMO wireless system with continuous phase modulation. The presented MIMO CPM receiver separates the received mixtures into the transmitted CPM signals without knowledge of the MIMO channel. The separated signals are demodulated with incoherent CPM demodulators. The presented MIMO CPM system achieves a Bit Error Rate less than $1 \cdot 10^{-5}$ for a full response MIMO CPM (1RC) with a SNR higher than $25 \mathrm{~dB}$. In case of a carrier frequency mismatch the approach shows almost the same performance.

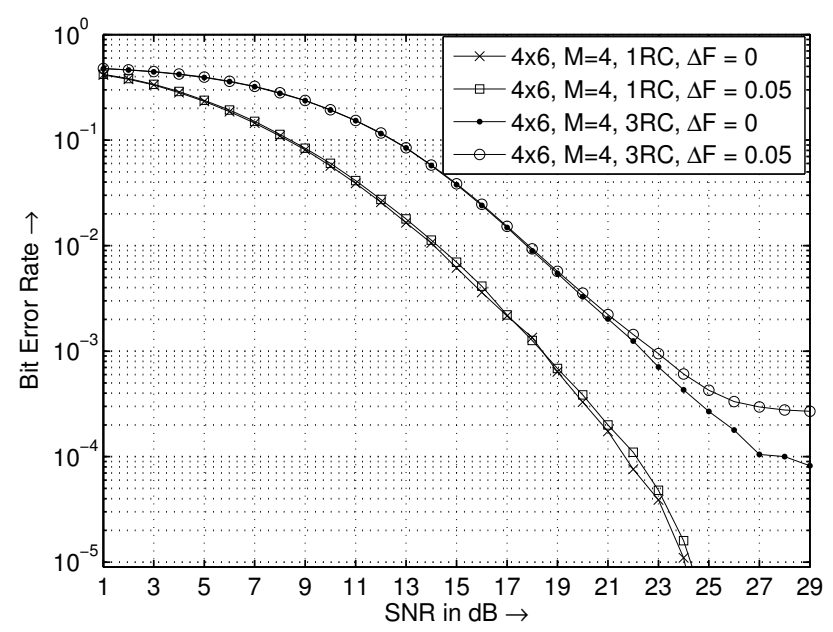

Fig. 5. Bit Error Rate (BER) versus the Signal-to-Noise-Ratio (SNR) for the MIMO CPM system with $N_{T}=4$ transmit and $N_{R}=6$ receive antennas.

\section{References}

Anderson, J. B., Aulin, T., and Sundberg, C.-E.: Digital Phase Modulation, Plenum Press, London, 1986.

Cardoso, J.-F. and Souloumiac, A.: Blind Beamforming for Non Gaussian Signals, in: IEE-Proceedings-F, 140(6), 362-370, 1993.

Hyvärinen, A., Karhunen, J., and Oja, E.: Independent Component Analysis, John Wiley \& Sons, New York, 2001.

Laurent, P. A.: Exact and Approximate Construction of Digital Phase Modulations by Superposition of Amplitude Modulated Pulses (AMP), IEEE Transactions on Communications, 34, 150$160,1986$.

Weikert, O. and Zölzer, U.: New Approach for Resolving Ambiguities for Semi-blind Equalization of MIMO Frequency Selective Channels, in: Proc. of 17th Annual IEEE International Symposium on Personal, Indoor and Mobile Radio Communications (PIMRC'06), Helsinki, Finland, 2006.

Zhang, X. and Fitz, M.: Soft-Output Demodulator in Space-TimeCoded Continuous Phase Modulation, IEEE Transactions on Signal Processing, 50, 2589-2598, 2002.

Zhang, X. and Fitz, M.: Space-Time Code Design with Continuous Phase Modulation, IEEE Journal on IEEE Journal on Selected Areas in Communications, 21, 783-792, 2003.

Zhao, W. and Giannakis, G.: Reduced Complexity Receivers for Layered Space-Time CPM, IEEE Transactions on Wireless Communications, 4, 574-582, 2005. 